\title{
How are students' ability and perception in using media through synthesis organic chemistry learning?
}

\author{
Lusia Narsia Amsad* \\ Post Graduate Chemistry Education \\ Universitas Cenderawasih \\ Jayapura, Indonesia \\ lusianarsiaamsad@gmail.com
}

\author{
S Liliasari, A Kadarohman, R E Sardjono \\ Post Graduate Chemistry Education \\ Universitas Pendidikan Indonesia \\ Bandung, Indonesia
}

\begin{abstract}
Lecturer usually uses media in order to help students' understanding about organic chemistry. In this research, the aim is to find out the ability and perception of students in learning by using media in the form of online and offline. The offline media we used was namely Spartan Student V6, and ChemAxon Reactor software, while the online media used is Reaction Predictor. Participants from this study were 16 students from one of the state universities in Papua. This research is a descriptive research by using the percentage of data and from questionnaire results. It shows the ability of students' in using media.. Based on the results of this study, it was found that the ability of students in using mediathrough the learning of organic synthesis was still low, around $10-40 \%$. The media that highest used is Reaction Predictor onlinemedia and the lowest used is Spartan Student V6 offline media. It was also known that the results of students' responses for using media in learning synthetic organic chemistry are positive.
\end{abstract}

Keywords: The use of media, Students'ability and perception, Learningof organic synthesis

\section{INTRODUCTION}

The use of media in learning organic chemistry is currently growing rapidly. Based on previous research organic chemistry learning uses media to help students in analyzing answers by predicting reactants, reagents or products [1,2]. Media used to predict reactions of organic complex compounds or target compounds. Media used to visualize the structure of compounds in the form of 2D-3D $[3,4]$. It also can show the reaction mechanism from starting material into a complex molecule. The media is also can support students to be actively involved and responsible in the learning process $[5,6,7,8]$.

The media used can be a Clickers program. This media makes it possible to analyze student answers in retrosynthetic analysis, so that students can develop problem solving skills [8]. Through the use of OrganicPad media which is an innovative tablet computer based media.
Using media in organic chemistry learning assists students visualize the stages of the reaction mechanism and help students to be mechanistic thinking [9].

The development of technology makes the help of studying mechanistic reactions through the estimation of interactions between molecular orbitals, therefore a learning machine can be used. The Learning Reaction Predictor engine is used to predict complex chemical reactions $[4,10]$. In addition, we can use ChemAxon reactor in learning media that can develop ability of students in predicting reactions. In addition to knowing molecular integrity, students can use the Spartan Student v6 software media in the learning of synthetic organic chemistry [11-15].

The purpose of this study is to determine the ability and perception of students in learning by using media in the form of online and offline. The media used is 3 (three) types of media. Offline media is the Spartan Student V6 software, and ChemAxon Reactor Software, while the online media used is Reaction Predictor. These three media are used in learning organic synthesis [13].

\section{Materials AND Method}

Descriptive method is the method used in this study. In this case the description is the result of the perception and the results of students using learning software. The research participants were 16 students from public universities in Papua. They were students in the fourth year. The student has attended lectures on organic chemistry 1,2 and advanced organic chemistry.

There are two media used in this study, namely offline and online media. The offline media used is the Spartan Student V6 software and ChemAxon reactor software as shown in Figure 1 


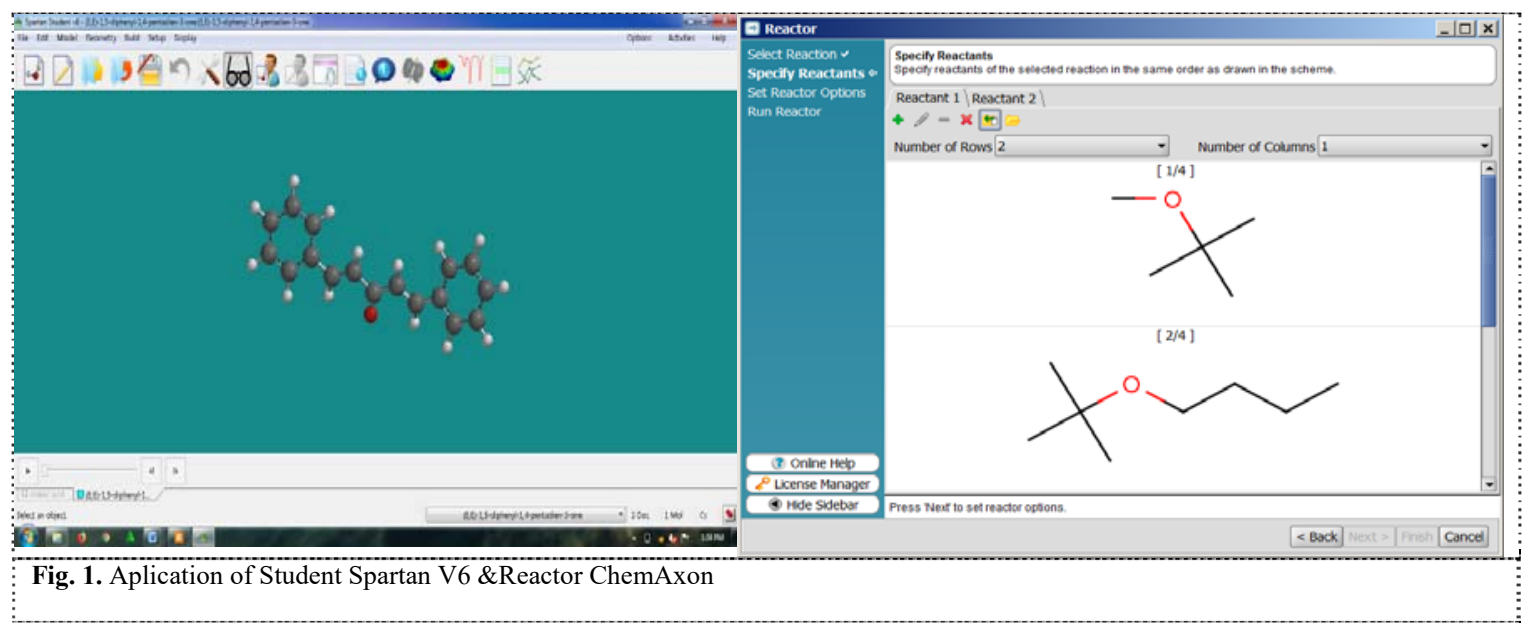

The Spartan Student V6 software is included in an interface that makes 3D structures for organic, inorganic and organometallic compounds. Student Spartan V6 media also has the ability to display 2D sketches for organic molecules, polypeptides, polynucleotides, and has procedures for guessing transition states [13]. As with the ChemAxon Reactor application, it provides a practical way to process virtual reactions quickly, resulting in compounds that have been registered; In addition, reactions that contain rules defined in chemical terms can provide products that are synthetically feasible [11].

In online media in the form of Reaction Predictor as in figure 2.Reaction Predictor application is one part of the http://cdb.ics.uci.edu webserver that is run online that attempts to overcome the problem of predicting the reaction mechanism depending on the reagents and conditions.

The webserver is an overall implementation of the synthesis approach of organic compounds using learning software to predict reactions $[4,10]$. The results of students in using these three media will be analyzed, to determine the ability of students to use the media. In addition, the results of student questionnaires will also be analyzed to find out their perceptions of the use of media in learning organic synthesis.

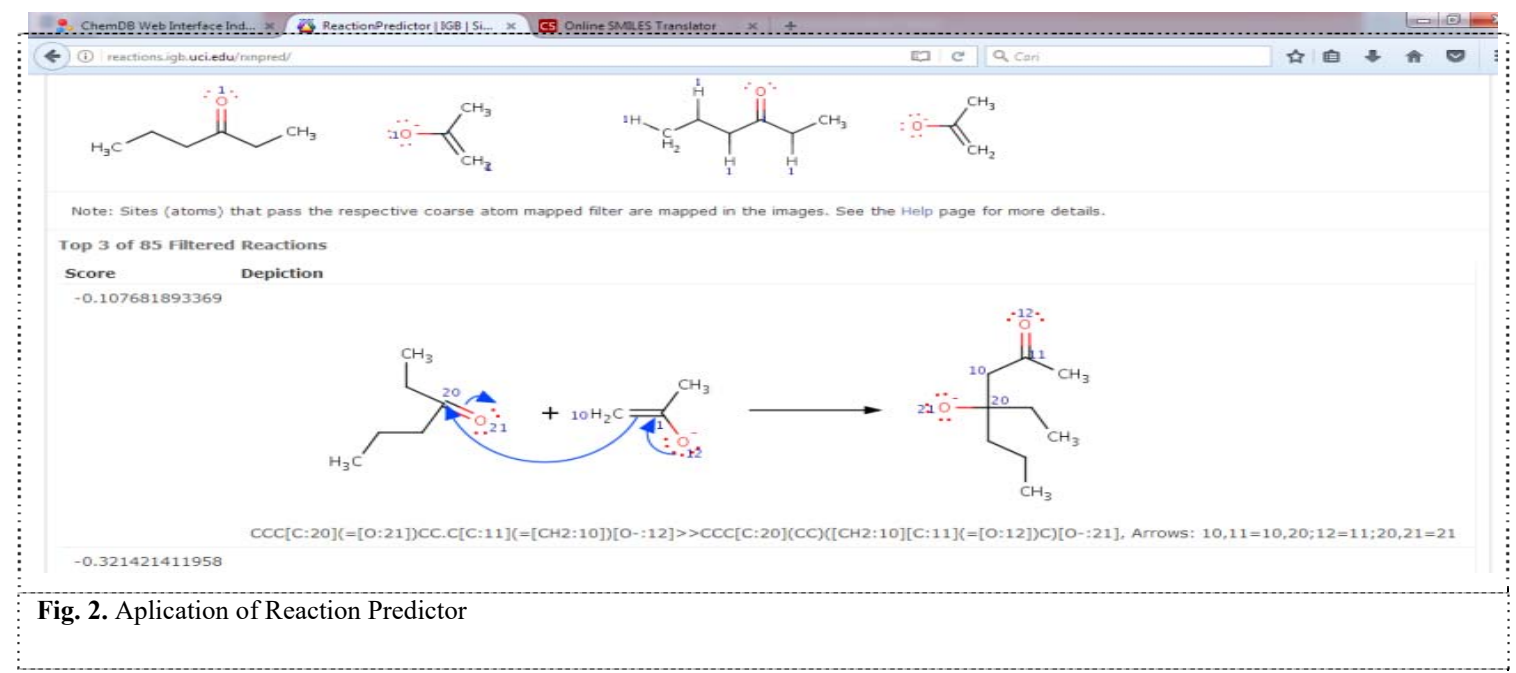




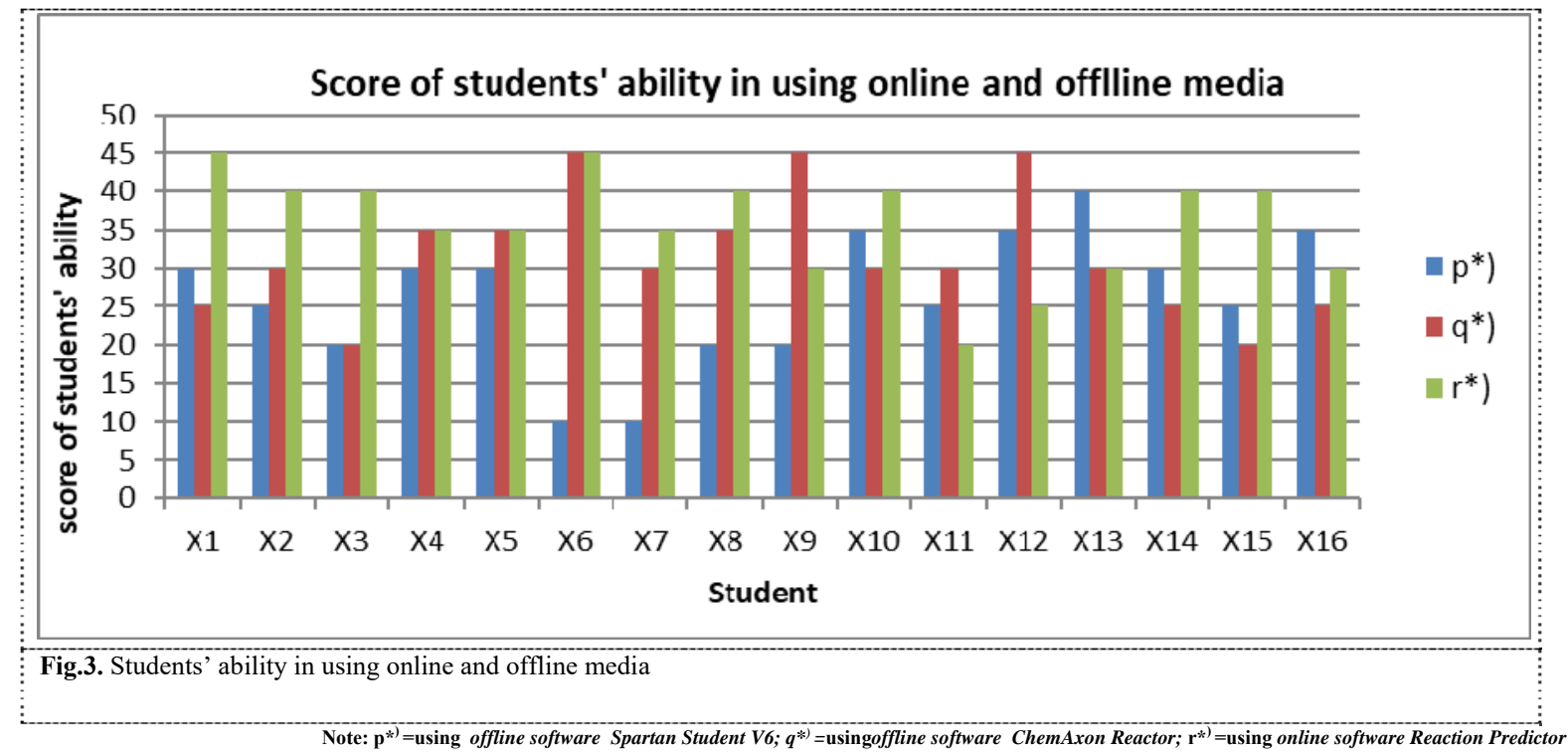

\section{RESULTS AND DISCUSSION}

It has been argued that the importance of a very complex synthetic organic chemistry learning process is supported by a learning medium. The use of media in learning organic synthesis in this study is expected to improve students' thinking skills. Based on the results obtained by students in all three uses of this media can be seen in Figure 3.

Based on the results of the use of media above, it can be seen that the results of students' ability to use media still very low at around $10-40 \%$. This may be because students are not used to and are still learning to use the media in learning synthetic organic chemistry. In the use of offline media in the form of Student Student V6 software, the results are lower than the others. This may be due to students being given only limited time to operate this offline media and only in groups. In this case students experience difficulties in answering questions related to the tasks assigned. Students use the Spartan Student V6 to predict the stability of compounds from geometry isomers, compound conformations, and geometric structures. Associated with the prediction of target molecular compounds and the starting material reacted in this study.
Students use of ChemAxon and Reaction Predictor Reactor software which is an online media,to see the predictions of various possible compounds starting material from a specific reaction that can produce molecular target compounds. Besides knowing how the starting reactions of the material are in specific conditions. In the ChemAxon Reactor software students are given the opportunity to be able to install and operate on their respective laptops or in groups. But the assignments given to be completed individually outside of learning activities turned out to only help a few students in completing the existing tasks, and in general the answers given by students were the same; so that there is a tendency for them to do it together or just see from the answers of their friends. However, it is different from the results of students' ability to use Reaction Predictor online media which looks much better because of how to operate it that can be online. Even students can operate it not only using a laptop/notebook or on a computer, but can use their smartphone. This resulted in them being able to continue to train their abilities, so the results were far better.

In addition, it can be seen the response of students to the use of the media used, in the learning of synthetic organic chemistry. The results can be seen in Figure 4. 


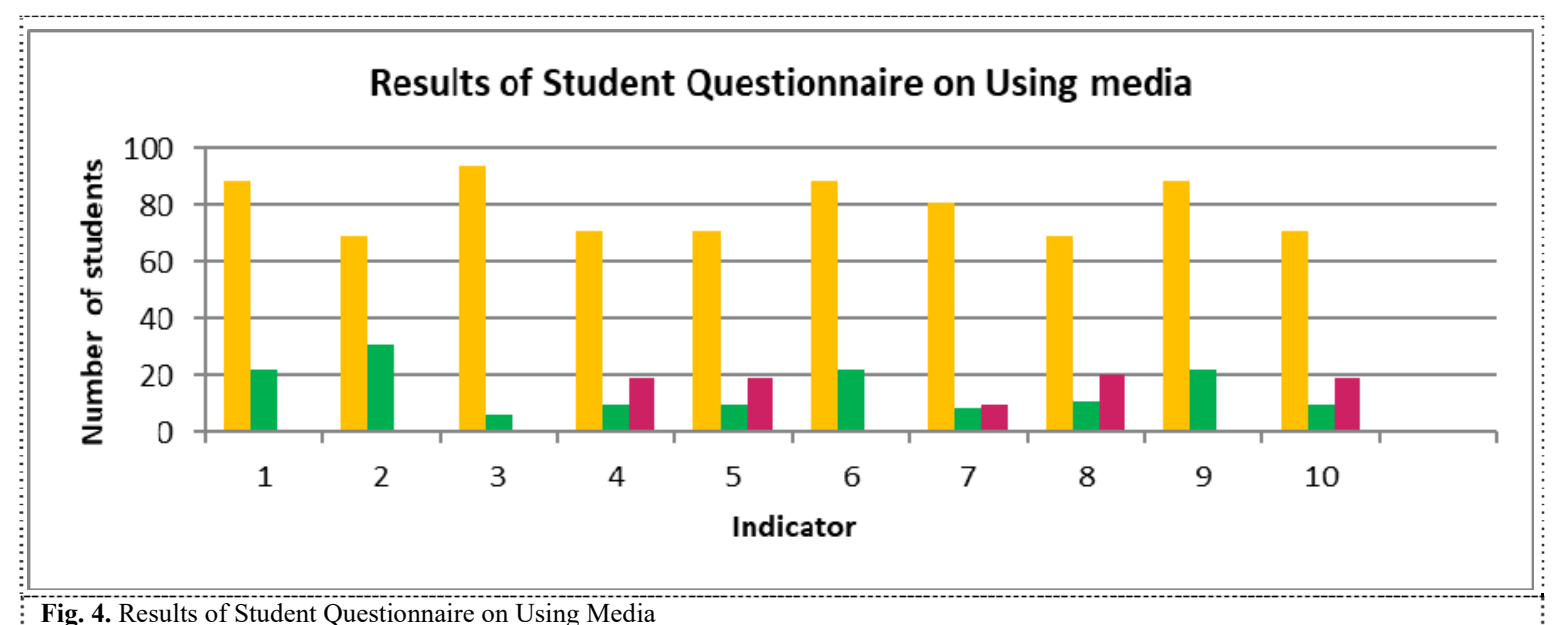

Fig. 4. Results of Student Questionnaire on Using Media

Based on Figure 4, it is known that the indicators of the questionnaire are that the use of media consists of: 1) train students to design their own experiments to solve the problem of synthesis of organic compounds; 2) provide opportunities for students to be able to submit hypotheses related to the analysis of retro-synthesis of organic compounds; 3 ) provide students new ways to solve the problem of synthesis of organic compounds; 4) collaborate students in groups; 5) making students learning challenging and fun; 6) training students' HOTS; 7) explore students' opinions in discussions through this learning media, 8) students be more responsible for the tasks given in lectures with this learning media; interesting because using ICT media; 10) students understanding lecture material with this learning.

It can be seen that overall the results obtained by students showed a positive result in the use of the media. The results of the students' agreement on the use of media were around $70-95 \%$. This shows that the use of media in learning organic synthesis have a good impact on students and also increase learning outcomes for the courses.

Wherever it is associated with the results of previous studies, it can be seen that the use of media for students improve the ability of collaboration and communication between students in groups. Even the ability of self-directed learning also improve through the use of media in learning and enable the creation of more conducive learning situations $[16,17]$. HOTS of students can also be stimulated through the use of media in classroom learning [5].

\section{CONCLUSION}

From the results of this study, it is known that the ability of students to use media in the learning of synthetic organic chemistry is still low at around $10-40 \%$. The highest media usage results are found in online Reaction Predictor media compared to the use of ChemAxon Reactor software and Reaction Predictor. The offline media in the form of Spartan Student V6 software is the lowest. But the results of student responses to the use of media in the learning of synthetic organic chemistry are positive, as seen from the respondents who agreed to use it around $70-95 \%$. For this reason, the lecturers need attention to facilitate the use of media in the learning of synthetic organic chemistry.

\section{ACKNOWLEDGEMENT}

We wish to thank all the team of organic lecturers and laboratory assisted at one of the State Universities in Papua who have helped in data collection.

\section{REFERENCES}

[1] Grove N P, Cooper M M and Rush K M. "Decorating with Arrows: Toward the Development of Representational Competence in Organic Chemistry," 2012.

[2] Flynn A B. "Developing Problem-Solving Skills through Retrosynthetic Analysis and Clickers in Organic Chemistry,", 2011, 1496-500.

[3] Rens L Van, Dijk H Van, Mulder J and Nieuwland P. "Using a Web Application To Conduct and Investigate Syntheses of Methyl Orange Remotely," 2011.

[4] Kayala M A and Baldi P. "Reaction Predictor: Prediction of complex chemical reactions at the mechanistic level using machine learning $J$. Chem. Inf". Model, 2012, 52 2526-40.

[5] Bhattacharyya G."From Source to Sink: Mechanistic Reasoning Using the Electron-Pushing Formalism J. Chem. Educ.90" 1282-9. 2013.

[6] Online V A. "Research and Practice understanding of 2D - 3D diagrammatic relationships $\dagger, 2013,177-87$.

[7] Angelo J G D. "Use of Screen Capture To Produce Media for Organic Chemistry," 2014.

[8] Flynn A B " Research and Practice How do students work through organic synthesis learning activities? Chem. Educ. Res. Pract.15,", 2014, 747-62.

[9] Grove N P and Lowery Bretz S. "A continuum of learning: from rote memorization to meaningful learning in organic chemistry Chem. Educ. Res.", 2012, Pract.13 201.

[10] Warr W A. "A short review of chemical reaction database systems, computer-aided synthesis design, reaction prediction and synthetic feasibility Mol. Inform.33", 2014, 469-76.

[11] Mcr S I, Kalinski C, Umkehrer M, Weber L, Kolb J, Burdack C, Ross G, Ag P and Straße G. "On the industrial applications of MCRs: molecular diversity in drug discovery and generic drug synthesis," 2010, 513-22. 
[12] Ealy J B."A Student Evaluation of Molecular Modeling in First Year College Chemistry 8," 1999.

[13] Clauss A D and Nelsen S F Integrating Computational Molecular Modeling into the Undergraduate Organic Chemistry Curriculum 86 955-8

[14] Venkataraman B."Visualization and interactivity in the teaching of chemistry to science and non-science students, 2009, 62-9.

[15] Davis R A,"Organic \& Biomolecular Chemistry 10,"2012.

[16] Parker L L and Loudon G M,"Case Study Using Online Homework in Undergraduate Organic Chemistry: Results and Student Attitudes," 2013.

[17] Song Lyan \& Hill J R, "A Conceptual Model for Undersatnding SelfDirected Learning in Online Environment J. Interact. Online Learn, 2007, 6 27-42. 